\title{
Abnormal Microspore Development Leads to Pollen Abortion in a Seedless Mutant of 'Ougan' Mandarin (Citrus suavissima Hort. ex Tanaka)
}

\author{
Zhiyong Hu, Min Zhang, Qigen Wen, Jie Wei, Hualin Yi, and Xiuxin Deng ${ }^{1}$ \\ National Key Laboratory of Crop Genetic Improvement, National Center of Crop \\ Molecular Breeding, Huazhong Agricultural University, Wuhan 430070, China \\ Xianghua Xu \\ Lishui Forestry Institute of Zhejiang Province, Lishui 323000, Zhejiang, China
}

\begin{abstract}
AdDitional INDEX words. male sterility, cytochemical, microsporogenesis, bud sport mutation
Abstract. Seedlessness is of commercial importance in citrus (Citrus L.). Seedless 'Ougan' mandarin (C. suavissima) was selected from a bud sport mutation that occurred in 'Ougan' mandarin. We analyzed their pollen viability through KI- $\mathrm{I}_{2}$ and FDA staining, and examined the anthers of wild-type (seedy) and seedless mutant 'Ougan' mandarin using histological and cytochemical methods to characterize the process of pollen development. No pollen fertility was detected in this mutant. Pollen abortion in anthers of the mutant occurred at the tetrad stage of microspore development, and almost all the tetrads were abnormal. The mutant had heterogeneous microspore populations, including monads, dyads, triads, tetrads, and polyads in the same microsporangium. Pollen grain number per anther of the mutant was $\mathbf{2 1 . 9 \%}$ less than the wild type. Morphology of mature pollen grains using SEM showed that the shape of mature pollen grains from both wild type and mutant is similar, but the microsporangia of the latter contained pollen grains of more variable sizes. At the early mature pollen grain stage, abundant starch grains and lipids appeared in the wild type's pollen, but fewer amounts were observed in the mutant. Moreover, the tapetal cells of the wild type accumulated lipids, but not those of the mutant. Results indicated that the abnormal development of the microspore led to pollen abortion in the mutant, and this could be the reason for its seedlessness. However, the genetic reasons for the aberrant tetrads are not clear and are under investigation.
\end{abstract}

'Ougan' mandarin is an ancient and peculiar Chinese native citrus cultivar, grown in the south of Zhejiang. It is a very tasty and easy-to-peel mandarin, cultivated for over 1000 years in China. Additionally, it has good shelf life at room temperature and has been considered to have medicinal application because its fruit are rich in vitamins, flavonoids, monoterpenoids, etc. (Yang, 2002). The wild type is seedy with each fruit containing $\approx 10$ seeds. Seedlessness is an attractive trait for fresh fruit and a convenience for the consumer. In fact, many economically important commercial citrus cultivars worldwide are seedless. They originated from conventional breeding programs, from bud sport selections or chance seedling variation (Deng, 2005). The seedless cultivars from the bud sport mainly belong to three types: Satsuma mandarin (C. unshiu Marc.) (Malidzan et al., 2004), navel orange (C. sinensis Osbeck) (Yi et al., 2003), and Clementine mandarin (C. reticulata Blanco) (Bono et al., 1996; Russo, 2004). Each type includes dozens of cultivars with 4- to 6-month-long harvest periods. Seedless 'Ougan' mandarin is desired from a bud sport mutation found in Zhejiang Province in 1996. This mutant maintains all horticultural traits of the wild-type 'Ougan' mandarin.

Various researchers have achieved male-sterile materials by disrupting pollen development through induced mutation or genetic transformation (Daniell, 2002; Goetz et al., 2001; Guerineau et al., 2003). In citrus, with the aim of seedlessness,

Received for publication 28 Oct. 2006. Accepted for publication 2 June 2007. This research was financially supported by the National Natural Science Foundation of China (Grant no. 30471201) and the Ministry of Education (IRT 0548).

We thank Qiang Xu, Chunli Chen, and Wenwu Guo for their helpful suggestions. ${ }^{1}$ Corresponding author. E-mail: xxdeng@mail.hzau.edu.cn.
Li et al. $(2002,2003)$ introduced the chimeric ribonuclease gene into Ponkan mandarin (C. reticulata) and Valencia sweet oranges $(C$. sinensis) by agrobacterium [Agrobacterium tumefaciens (Smith \& Towns.) Conn. EHA105] transformation. Guo et al. (2004) conducted somatic fusion of embryogenic suspension-derived protoplasts of Satsuma mandarin, a male-sterile cultivar, with leaf protoplasts of seedy types of citrus, in an attempt to generate seedless cybrids. Male sterility in citrus generally leads to seedlessness because of the parthenocarpy characteristic of most citrus genotypes.

Knowledge of male sterility in citrus is limited because woody plants have long life cycles, making them a difficult group to study. Yamamoto et al. $(1993,1995)$ investigated the relationship between male sterility and seedlessness in citrus and found that seedlessness in diploid citrus generally was related to male sterility or to self-incompatibility. They reported that cytoplasmic male sterility in $C$. unshiu was due to nuclear-cytoplasmic interaction (Yamamoto et al., 1997). Furthermore, Hu et al. (2006) reported that the timing and cellspecificity of mitochondrial gene expression were differentially regulated during male gametophyte development in C. unshiu and suggested that this was probably associated with its pollen abortion. In 'Ougan' mandarin, little evolution about male sterility has been conducted. Zeng et al. (2005) reported that rare, naturally released pollens and difficulty in pollen germination were responsible for the seedlessness of the mutant 'Ougan' mandarin. However, the development of mature pollen grains from the microspore mother cell (MMC) in the seedless mutant 'Ougan' mandarin is unknown.

In the current study, the development of anthers of both the mutant and the wild-type 'Ougan' mandarin were studied 
to determine the onset and structural manifestations of pollen abortion, with particular attention to the distribution of polysaccharides, lipids, and callose during anther development.

\section{Materials and Methods}

Plant materials. Flower buds were collected from an 8-year-old citrus orchard located in Lishui County, Zhejiang Province, China. The flower buds of the mutant (seedless) were from the clonal multiplication trees top-grafted to Satsuma mandarin as interstock and trifoliate orange [Poncitrus trifoliate (L.) Raf.] as rootstock. The wild type (seedy) was grafted on trifoliate orange. Flower buds from three trees both for wild-type and mutant plants of 'Ougan' mandarin were collected at different stages to observe microspore development from the early premeiotic stage to mature pollen.

Pollen Viability ANd Grain number Per ANTHER. Ten flower buds collected from three trees before anthesis were sampled, all the pollens were freshly harvested. For pollen stain viability, one anther per flower bud was collected from six flower buds, mixed, and spread on 10 slides, and then three observations per slide were completed, including about 100 pollen grains each. Thirty observations per genotype were carried out. Two stain methods were applied to analyze pollen viability. In the first, the viable pollen grains were stained on the slide with $1 \%$ iodine-potassium iodide $\left(\mathrm{KI}-\mathrm{I}_{2}\right)$, and the darkness of the staining was used as an indicator of pollen fertility. The second stain test consisted of a drop of fluorescein diacetate (FDA) solution $\left(100 \mu \mathrm{g} \cdot \mathrm{mL}^{-1}\right)$ was added to the pollen on the slide. After staining for $5 \mathrm{~min}$, pollen grains were observed at WIBA wavelength using a BX61 fluorescence microscope (Olympus, Tokyo). It was assumed that the intense fluorescence is correlated with viable pollen (Liu et al., 2004). To count the number of pollen grain per anther, 100 anthers were collected from at least 10 flower buds, crushed, and mixed in $25 \mathrm{~mL}$ of distilled water containing two drops of surfactant (Tween-20, Amresco, Solon, $\mathrm{OH}$ ). Counts were made in at least on 10 hemocytometer fields to calculate the average number of pollen grains per anther (Chen et al., 2004).

Histological analysis. Both the wild-type and the mutant flower buds were fixed overnight in 5 formalin : 5 acetic acid : 90 alcohol [FAA (by volume)] at $4{ }^{\circ} \mathrm{C}$. Fixed flower buds were dehydrated using a graded ethanol series $(50 \%, 70 \%, 95 \%$, and $100 \%$ ) and embedded in Technovit 7100 resin (Heraeus, Kulzer, Germany). Transverse sections $\approx 1 \mu \mathrm{m}$ thick were cut from the embedded blocks using a Leica Ultracut $\mathrm{R}$ ultramicrotome (Leica, Bensheim, Germany). The sections were stained with $2 \%$ Toluidine Blue O (Aldrich, Milwaukee) solution for cytological observation. Several anthers fixed in FAA were squashed and stained with $1 \%$ acetocarmine in $45 \%$ acetic acid to observe microsporocyte morphology.

Polysaccharide, lipid, and CAllose localization. To detect insoluble polysaccharides, anther sections were oxidized for $10 \mathrm{~min}$ in $0.5 \%$ periodic acid in $0.3 \%$ nitric acid and rinsed in running water for 1-2 min, with a final rinse in distilled water. After staining for $15 \mathrm{~min}$ in Schiff's reagent, the sections were washed three times in $0.5 \%$ sodium metabisulfite for 2 min each, rinsed for $5 \mathrm{~min}$ in running water, and transferred to distilled water. To detect lipids, anther sections were rinsed for $1-2$ min in $70 \%$ ethanol, stained in fresh $1 \%$ Sudan Black B in $70 \%$ ethanol for $30-60 \mathrm{~min}$ at $50-60{ }^{\circ} \mathrm{C}$, rinsed for $1 \mathrm{~min}$ in $70 \%$ ethanol, and transferred to distilled water (Xie et al.,
2005). For callose, sections were stained with $0.01 \%(\mathrm{w} / \mathrm{v})$ Aniline Blue in $0.1 \mathrm{M}$ phosphate buffer, pH 9 (Zhang and Fernando, 2005).

All observations were performed under a light BX-61 fluorescence microscope. All photographs were taken using an Olympus DP 70 CCD camera.

Mature pollen grain morphology. The FAA-fixed anthers were dehydrated using a graded ethanol series $(50 \%$, $70 \%$, and $95 \%$ ) and degreased by treating them twice, $20 \mathrm{~min}$ each, with xylene. This was followed by critical-point drying, mounting on copper stubs, and sputter coating with gold. The samples were examined under a Quanta 200 scanning electron microscope (FEI Co., Hillsboro, OR), and representative images were obtained.

\section{Results}

Pollen Viability AND Grain nUmber Per anther. Stainability by $\mathrm{KI}-\mathrm{I}_{2}$ reflects the amount of starch accumulated in the pollen and is highly correlated with pollen fertility (Liu et al., 2004). In general, darkly stained pollen grains are regarded as viable (Fig. 1A), whereas those lightly stained or unstained are nonviable (Fig. 1B). About $84.5 \%$ of the pollen of the wild type was darkly stained, but none of the mutant's pollen was stained. Stainability with FDA mediated by the activity of esterase in the pollen also verified this difference in pollen fertility. For the wild-type plant, FDA staining accounted for $71.4 \%$ of the pollen (Fig. 1C), whereas the mutant pollen was again not stained (Fig. 1D). Thus, the wild type clearly has high pollen fertility, as assayed by both $\mathrm{KI}-\mathrm{I}_{2}$ and FDA staining, and the mutant has absolutely no viable pollen.

Pollen grain number per anther of the wild type and the mutant were 13,130 and 10,250; respectively. Mutant anther contained $21.9 \%$ less pollen grains per anther than wild type.

Pollen development. Before meiosis, MMCs in the anther locule were spherical or elliptical, arranged next to each other

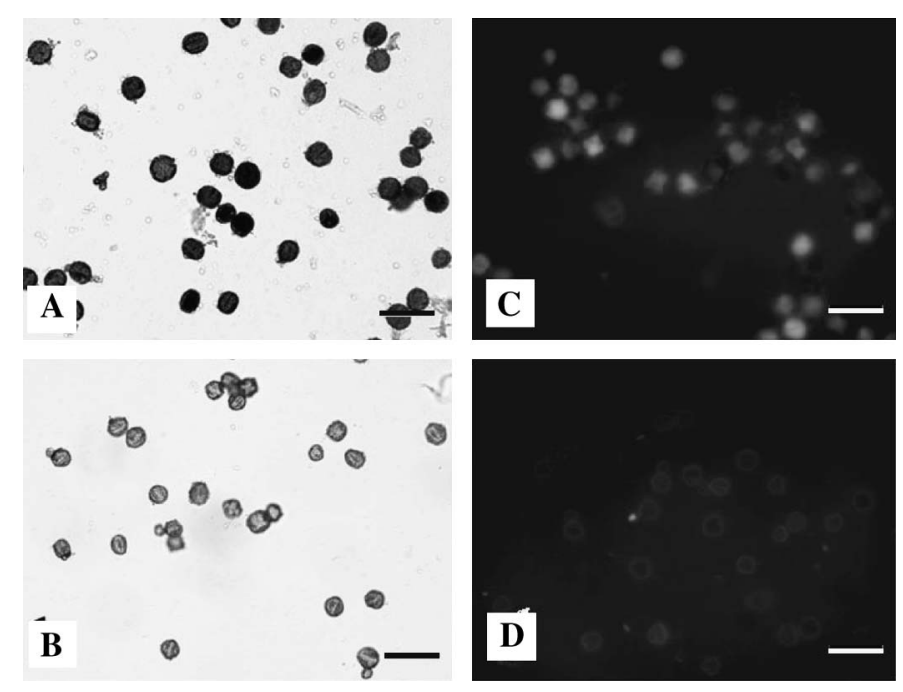

Fig. 1. Pollen viability of 'Ougan' mandarin. (A) Mature pollen grains of the wild type stained with iodine-potassium iodide $\left(\mathrm{KI}^{-} \mathrm{I}_{2}\right)$ showing high pollen viability. (B) Mature pollen grain of the mutant stained with $\mathrm{KI}_{2} \mathrm{I}_{2}$ showing that all pollens were sterile. (C) Mature pollen grains of the wild type stained with fluorescein diacetate (FDA) showing that most of the pollen had high activity. (D) Mature pollen grains of the mutant stained with FDA showing no pollen activity. (Scale bars indicate $50 \mu \mathrm{m}$.) 
without any intercellular spaces, with prominent, centrally located nuclei (Fig. 2A). Dark wall material around the MMCs indicated callose deposition. At this stage, the anther wall differentiated into four layers: epidermis, endothecium, middle layer, and tapetum, each differing in morphology. The innermost layer of the anther wall was the tapetum, containing cells that were large and square-shaped, with dense cytoplasm and one or two nuclei per cell.

After MMC meiosis, tetrad cells were surrounded by a dense callose wall (Fig. 2B). Tetrads of similarsized microspores were observed by squashing and stained with acetocarmine (Fig. $3 \mathrm{H}$ ). At this stage, tapetal cells became compressed and the cytoplasm began to concentrate.

Microspores released from the tetrads comprised of densely cytoplasmic cells with a centrally located nucleus (Fig. 2C). Tapetal cells appeared to enlarge with the filling of the cytoplasm.

Microspores became progressively more vacuolated and displaced the microspore nucleus to a peripheral position (Fig. 2D). The internal organization and external morphology of nearby tapetal cells became increasingly irregular. Some tapetal cells showed cavities, and others collapsed. The other three anther wall layers remained highly vacuolated and continued their differentiation. Pollen grains became densely cytoplasmic as they matured (Fig. 2E). Tapetal cells degenerated and were absorbed by pollen grains.

Mutant pollen development. The early development of the mutant anthers was essentially identical to those of the wild type during the MMC stage (Fig. 2F) and tetrad stages (Fig. 2G). After MMC meiosis in the mutant, however, variablesize microspores developed and were released from the tetrads (Fig. $2 \mathrm{G}$ and $\mathrm{H}$, arrowheads).

Observations after squashing and staining with acetocarmine revealed that the mutant had heterogeneous microspore populations including monads, dyads, triads, tetrads, and polyads in the same microsporangium (Fig. 3A-G). Tetrads of similar-sized microspores were rarely seen, and most of them consisted of microspores of different sizes.
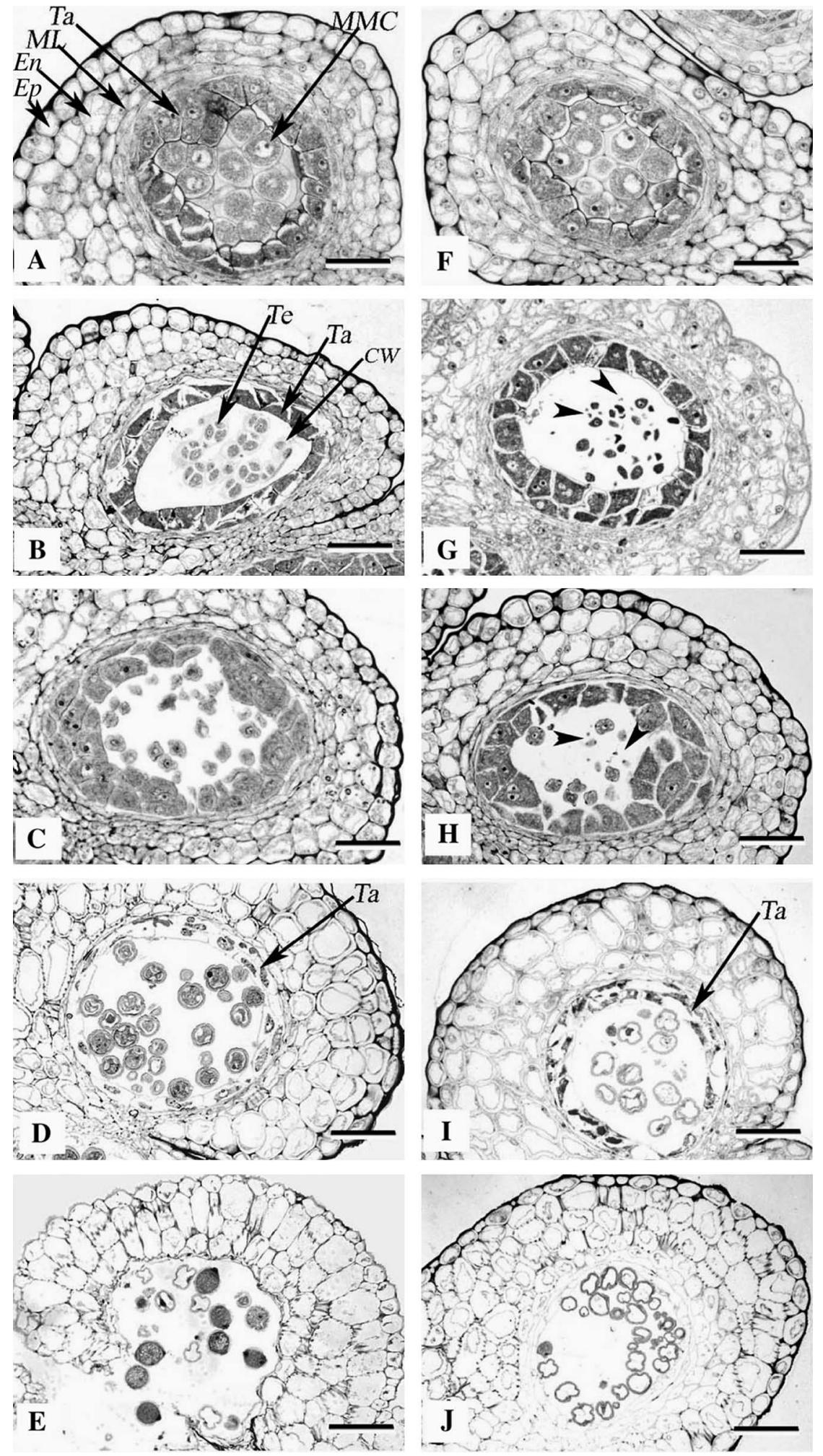

Fig. 2. Micrographs of anther and pollen development in the wild type (A-E) and in the mutant (F-J) 'Ougan' mandarin stained with Toluidine Blue O. (A) Microspore mother cell (MMC) stage; each anther lobe consisted of epidermis, endothecium, middle layer, tapetum, and MMCs. (B) Tetrad stage, callose walls surrounded the microspore. (C) Early microspore stage showing the microspores separated. (D) (E) Early mature pollen grain stage showing the degenerating tapetum. (F) MMC stage. (G) Tetrad stage showing abnormal tetrad (arrowhead). (H) Early microspore stage showing many small microspores (arrowheads). (I, J) Mature pollen grain stage and tapetal cells almost completely disintegrated. $\mathrm{CW}=$ callose wall, $\mathrm{Ep}=$ epidermis, En $=$ endothecium, $\mathrm{ML}=$ middle layer, $\mathrm{Ta}=$ tapetum, $\mathrm{Te}=$ tetrad. (Scale bars indicate $30 \mu \mathrm{m}$ in A and $\mathrm{F} ; 40 \mu \mathrm{m}$ in B and $\mathrm{G} ; 50 \mu \mathrm{m}$ in $\mathrm{C}$ and $\mathrm{H}$; and $60 \mu \mathrm{m}$ in $\mathrm{D}, \mathrm{E}, \mathrm{I}$, and $\mathrm{J}$.) 

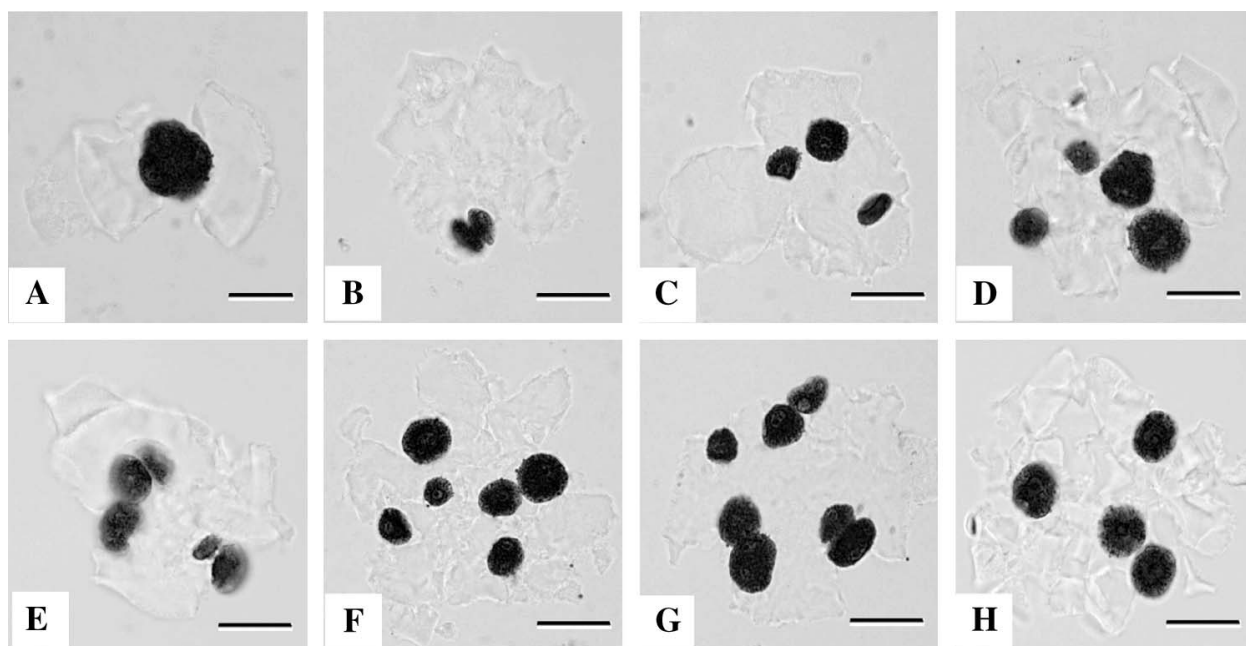

presence and localization of callose using Aniline Blue. Callose appeared bright blue when observed under a fluorescence microscope using ultraviolet (UV) excitation. At the MMC stage, callose was detected in both types (Fig. 5A and B). During the course of meiosis, the callose moved gradually toward the division side of the cytoplasm. Consequently, the MMC subdivided with the formation of callose into four parts in the wild type (Fig. 5C). However in the mutant, the MMC divided into varying parts with the formation of the callose (Fig. 5D).

MORPHOLOGY OF MATURE POLLEN USING SEM. Mature pollen grains from the wild type were spherical and $18-22 \mu \mathrm{m}$ in diameter (Fig. 6A). Similar shape of mature pollen grains were observed for the mutant, but it displayed a wider range of diameters,

In abnormal pollen development, microspores cytoplasm became less dense and shrunken, with the tapetum becoming vacuolated and degenerated (Fig. 2I). Subsequently, tapetal cells degenerated and disappeared, leaving various-sized aborted microspores without cytoplasm (Fig. 2J).

ChANGES IN POLYSACCHARIDE DISTRIBUTION DURING ANTHER DEVELOPMENT. No evident differences occurred in starch distribution between the wild-type and the mutant anthers before or during the $\mathrm{MMC}$ meiosis. During anther development of the wild type and the mutant, the distribution of polysaccharides in the anther correlated with the different stages of male gametophyte development.

During meiosis, starch grains accumulated in cells of the epidermis, endothecium, and middle layer of the anther wall (Fig. 4A-D), but only minor red coloring appeared in tapetal cells in the tetrad stage (Fig. 4C and D). The tetrad was labeled red, indicating the presence of insoluble polysaccharides in the microspores, but no red appeared in the callose (Fig. 4C and D).

During microspore development, the starch grains in the anther wall decreased markedly in abundance and disappeared from the anther (Fig. 4E and F). As pollen grains accumulated storage material, abundant starch grains appeared in wild-type pollen (Fig. 4E), but few in the mutant pollen (Fig. 4F), indicating the importance of starch grains, which serves as an energy material in both pollen and seed. Tapetal cells did not display any red color, suggesting a low abundance of polysaccharidic materials in these cells at any stage.

Changes of LiPID DURING POllen DEVELOPMENT. Before and during MMC meiosis, no lipid occurred in the anthers of both types (Fig. 4A-D). After meiosis, however, pollen grains began to accumulate lipids in both types. In the mature pollen grains of the wild type, abundant lipids were deposited at the surface of the pollen wall (Fig. 4E). At this stage, abundant lipids accumulated in the tapetal cells of wild-type anthers. But in the mutant anther, the surface of pollen grains contained very little lipid material and the tapetal cells contained no lipid at this stage (Fig. 4F).

DistRIBUTION OF THE CALLOSE DURING ANTHER DEVELOPMENT. Anthers at various stages of development were examined for the from 10 to $30 \mu \mathrm{m}$ (Fig. 6D). Looking from the polar front of mature pollen grains, we observed four colpos and many pores spread all over the pollen wall surface (Fig. 6B). Much lipid had accumulated on the surface of wild-type pollen, and this made the pollen surface look indistinct, although we degreased the samples
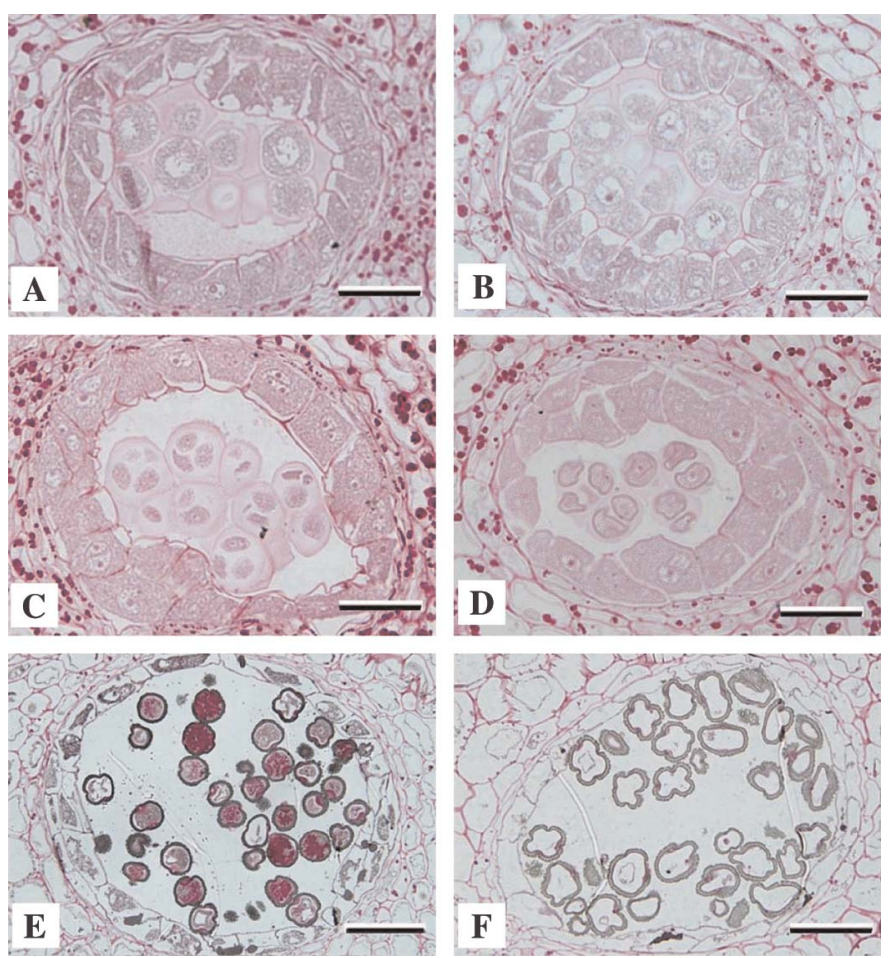

Fig. 4. Cytochemical analysis of pollen development in the wild type (A, C, E) and in the mutant $(\mathbf{B}, \mathbf{D}, \mathbf{F})$ of 'Ougan' mandarin. (A, B) Microspore mother cell (MMC) stage. Polysaccharide and starch stained red in anther walls. (C, D) Tetrad stage. Abundant starch occurred in anther walls. (E, F) Early mature pollen grain stage. Little of polysaccharide and starch stained in the anther walls. (E) In wild-type anthers, abundant starch and lipid material was present in the pollen grains. (F) In the mutant anther, pollen grains had less starch stained and lipid material. (Scale bars indicate $40 \mu \mathrm{m}$ in A and B; $50 \mu \mathrm{m}$ in C and $\mathrm{D}$; and $60 \mu \mathrm{m}$ in $\mathrm{E}$ and F.) 

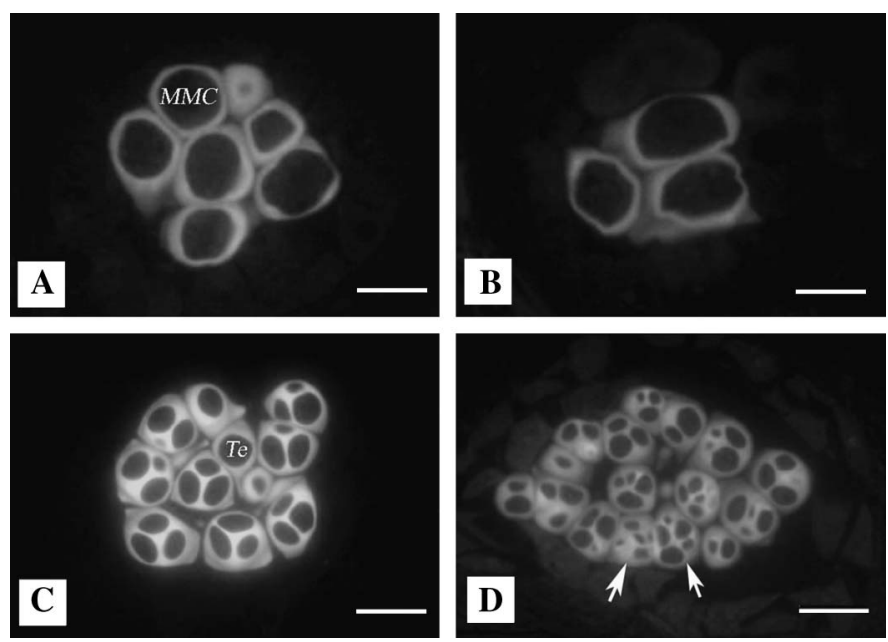

Fig. 5. Distribution of callose during the meiosis in the wild type $(\mathbf{A}, \mathbf{C})$ and in the mutant $(\mathbf{B}, \mathbf{D})$ of 'Ougan' mandarin. (A, B) Microspore mother cell $(\mathrm{MMC})$ at the early stage of meiosis. Note that callose accumulated along the cell walls and that the MMCs were enclosed in a thin callose wall. (C, D) Tetrad stage. (C) In wild type, the MMC divided into four parts with formation of the callose. (D) In the mutant, the MMC divided into varying parts with formation of the callose (arrow). (Scale bars indicate $60 \mu \mathrm{m}$ in A and $\mathrm{B}$ and $50 \mu \mathrm{m}$ in $\mathrm{C}$ and D.)

by passing them through xylene (Fig. $6 \mathrm{~B}$ and $\mathrm{C}$ ). In contrast, less lipid was observed on the pollen surface of the mutant, and it was clearer than the wild-type pollen surface (Fig. 6E and F).

\section{Discussion}

The seedlessness of the mutant 'Ougan' mandarin has attracted interests into the mechanisms of this trait. Rare, naturally released pollens and difficulty in pollen germination were proposed for the main reason of seedlessness in this mutant (Zeng et al., 2005). In this study, we found that in the 'Ougan' mandarin mutant, microspores could not develop into functional pollen. Although the shape of the mature pollen grains from the mutant was similar to that of pollen from the wild type, the aborted pollen displayed a wide range of diameters. Our results indicate that abnormal development of the microspore led to pollen abortion of the mutant. However, in the field, we observed that the mutant is seedy when it is grown alongside other citrus cultivars. This phenomenon indicates that the mutant is female fertile when cross-pollination takes place, therefore, suggesting that self-pollination with its own aborted pollen leads to seedlessness.

The onset of pollen abortion is critical to understanding the mechanisms for male sterility in higher plants (Xie et al., 2005). We have examined the processes of male gametophyte development in both wild-type and mutant 'Ougan' man- darin. In the present study, development of the male gametophyte was similar in both types until the formation of MMCs.

In the mutant, monads and polyads with various numbers and sizes of microspores were formed at the tetrad stage, suggesting meiotic abnormality in MMCs. Chen et al. (2004) also observed similar phenomena in citrus allotetraploid somatic hybrids and speculated that these phenomena resulted in the production of sterile pollens. Moreover, Hosoo et al. (2005) proposed that monads of Cryptomeria japonica (L. f.) D. Don was incited by nuclear fusion or defects in chromosome segregation. Formation of microspores from dyads to polyads seems to result from abnormal chromosome segregation and cytokinesis. Further investigation of the mutant is needed to discover the mechanism causing abnormal meiosis.

The difference in starch distribution between wild-type and the mutant anthers occurred in the early mature pollen grains, with abundant starch grains in wild-type pollen and few in the mutant. The result indicates the importance of starch grains as energy storage materials in pollen as well as seeds. The dynamics of the starch grains during microsporogenesis and pollen differentiation in higher plants have attracted wide attention. Starch grain deposition in microsporogenesis usually showed a regular distribution and a dynamic variation (Rodkiewicz et al., 1986). Singh (1978) concluded that the dense distribution of starch grains in the pollen is generally an important event during the regular development of a microspore. Lu et al. (2003) also reported that the starch grains highly accumulated in the fertile pollen of Picea asperata Mast. Another difference between the wild type and the mutant was in the degree of lipid accumulation in the tapetal cells especially in the surface of the pollen wall. At the early mature pollen grain stage, abundant lipids accumulated in the tapetal cells and the surface of the pollen wall of the wild type, but little accumulated in the mutant. Lipids might not be used by pollen as energy in 'Ougan' mandarin, perhaps as sticky material to adhere to the stigma. Another function of the large amount of
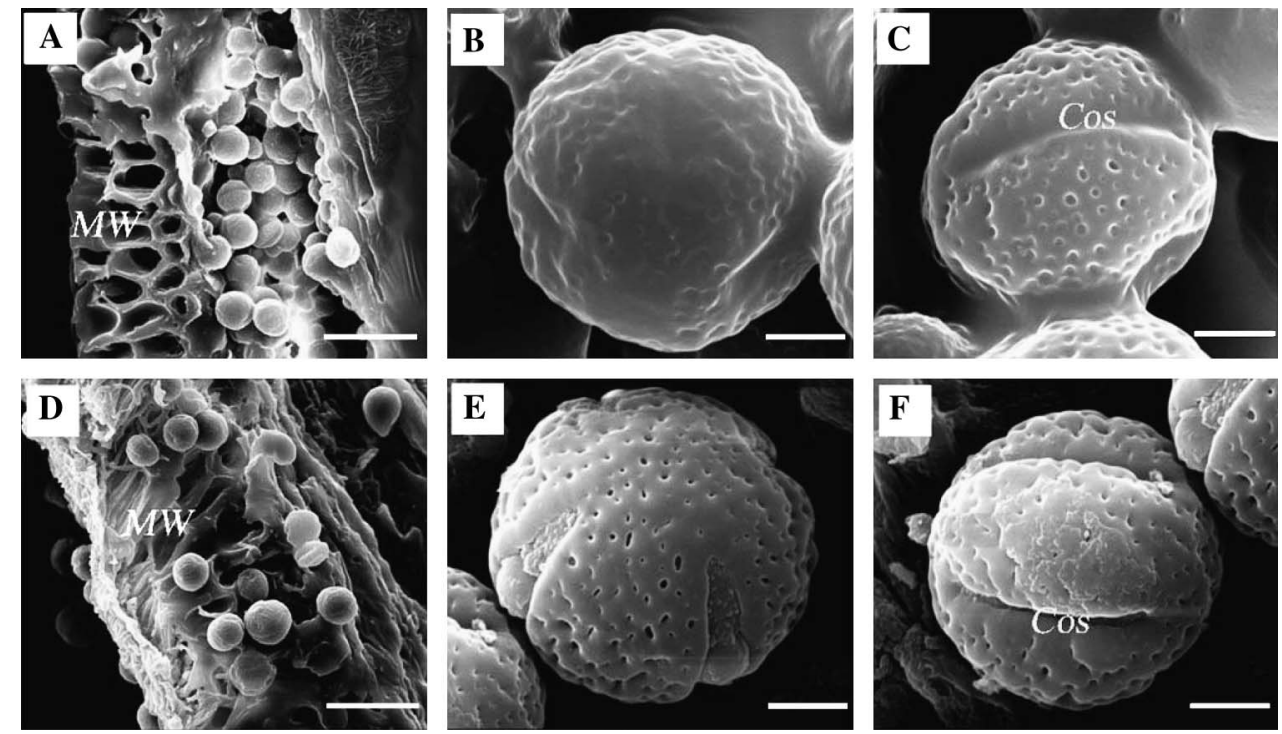

Fig. 6. Scanning electron micrographs of mature pollen grains in microsporangia of 'Ougan' mandarin. (A) Wildtype microsporangia containing uniformly sized pollen grains. (B) Wild-type mature pollen grain showing the polar front. (C) Wild-type mature pollen grain showing the equator front. (D) Mutant microsporangia containing pollen grains of varying sizes. (E) Mutant mature pollen grain showing the polar front. (F) Mutant mature pollen grain showing the equator front. $\mathrm{MW}=$ microsporangium wall, $\mathrm{Cos}=$ colpos. (Scale bars indicate $50 \mu \mathrm{m}$ in $\mathrm{A}$ and $\mathrm{D}$ and $5 \mu \mathrm{m}$ in $\mathrm{B}, \mathrm{C}, \mathrm{E}$, and F.) 
lipid accumulated in the surface of pollen wall is possibly the protection of pollen against ultraviolet light, which may inhibit the germination of pollen (Flint and Caldwell, 1984). No evident differences occurred in callose distribution between both types of anthers, suggesting that callose wall formation was unaffected by the developmental condition of the developing male gametophytes, consistent with the results of Zhang and Fernando (2005) in willow (Salix eriocephala Michx., $S$. exigua Nutt., S. purpurea L.).

Tapetal cells are physically the closest somatic cells to microspores, as they are positioned between male gametophytic tissues and surrounding sporophytic tissues. One function of tapetal cells is to synthesize lipidic materials to support microspore development (Ting et al., 1998; Xie et al., 2005). Many researchers have attributed male sterility in seed plants to deficiencies in the tapetum, which is intimately related with pollen nutrition (Aarts et al., 1997; Jin et al., 1997).

In the present study, both the wild-type and the mutant anther tissue appeared to have the same nutritional status, as reflected in cytochemical localizations of lipids and polysaccharides in the anther. At the early mature pollen grain stages, starch grains in the anther wall disappeared and lipid began to accumulate in the tapetum of wild-type anthers, suggesting a shift from polysaccharidic storage products to lipidic storage products in anther somatic tissue. However, little lipids accumulated in the mutant tapetal cells. The tapetum of wild-type anthers appeared to shift from polysaccharidic storage materials to lipid storage materials more effectively, as indicated by the accumulation of lipids in the surface of the pollen wall.

In citrus, genes involved in male sterility have rarely been studied. We examined the anthers of wild-type 'Ougan' mandarin and the seedless mutant using histological and cytochemical methods to characterize the process of pollen development. We expect that our current and future studies on 'Ougan' mandarin to contribute to the understanding of male sterility in citrus.

\section{Literature Cited}

Aarts, M.U., R. Hodge, K. Kalantidis, D. Florack, and Z.A. Wilson. 1997. The Arabidopsis male sterility 2 protein shares similarity with reductases in elongation/condensation complexes. Plant J. 12:615-623. Bono, R., J. Soler, and L. Fernandez de Cordova. 1996. 'Clemenpons' and 'Loretina,' two early clementine mandarin mutations of potential interest. In: B. Manicom (ed.). Proc. Intl. Soc. Citriculture, 8th Intl. Citrus Congr. 1:174-176.

Chen, C.L., W.W. Guo, H.L. Yi, and X.X. Deng. 2004. Cytogenetic analysis of two interspecific citrus allotetraploid somatic hybrids and their diploid fusion parents. Plant Breed. 123:332-337.

Daniell, H. 2002. Molecular strategies for gene containment in transgenic crops. Nat. Biotechnol. 20:581-586.

Deng, X.X. 2005. [Advances in worldwide citrus breeding]. Acta Hort. Sinica 32:1140-1146 (in Chinese).

Flint, S.D. and M.M. Caldwell. 1984. Partial inhibition of in vitro pollen germination by simulated soar UVB radiation. Ecology 65:792-795.

Goetz, M., D.E. Godt, A. Guivarch, U. Kahmann, D. Chriqui, and T. Roitsch. 2001. Induction of male sterility in plants by metabolic engineering of the carbohydrate supply. Proc. Natl. Acad. Sci. USA 98:6522-6527.

Guerineau, F., A. Sorensen, N. Fenby, and R.J. Scott. 2003. Temperature sensitive diphtheria toxin confers conditional male-sterility in Arabidopsis thaliana. Plant Biotechnol. J. 1:33-42.

Guo, W.W., D. Prasad, Y.J. Cheng, P. Serrano, X.X. Deng, and J.W. Grosser. 2004. Targeted cybridization in citrus: transfer of Satsuma cytoplasm to seedy cultivars for potential seedlessness. Plant Cell Rpt. 22:752-758.
Hosoo, Y., E. Yoshii, K. Negishi, and H. Taira. 2005. A histological comparison of the development of pollen and female gametophytes in fertile and sterile Cryptomeria japonica. Sex. Plant Reprod. 18:81-89.

Hu, Z.Y., Z. Tong, J. Wei, H.L. Yi, and X.X. Deng. 2006. Mitochondrial gene expression in stamens is differentially regulated during male gametogenesis in Citrus unshiu. J. Hort. Sci. Biotechnol. 81:565-569.

Jin, W., H.T. Horner, and R.G. Palmer. 1997. Genetics and cytology of a new genic male-sterile soybean (Glycine $\max$ L. Merr.). Sex. Plant Reprod. 10:13-21.

Li, D.D., W. Shi, and X.X. Deng. 2002. Agrobacterium-mediated transformation of embryogenic calluses of Ponkan mandarin and the regeneration of plants containing the chimeric ribonuclease gene. Plant Cell Rpt. 21:153-156.

Li, D.D., W. Shi, and X.X. Deng. 2003. Factors influencing agrobacterium-mediated embryogenic callus transformation of Valencia sweet orange (Citrus sinensis) containing the pTA29-barnase gene. Tree Physiol. 23:1209-1215.

Liu, H.Y., C.G. Xu, and Q.F. Zhang. 2004. Male and female gamete abortions, and reduced affinity between the uniting gametes as the causes for sterility in an indica/japonica hybrid in rice. Sex. Plant Reprod. 17:55-62.

Lu, S., Y. Li, Z. Chen, and J. Lin. 2003. Pollen development in Picea asperata Mast. Flora 198:112-117.

Malidzan, S., M. Radulovic, B. Lazovic, and T. Perovic. 2004. Derivation of early-ripening selection from a heterogenous clonal cultivar Kawano wase (Citrus unshiu Marc.), p. 107. In: E. Mohamed (ed.). Program and Abstract, Intl. Soc. Citriculture, 10th Int. Congr., Agadir, Morroco (abstr.).

Rodkiewicz, B., J. Bednara, A. Mostowska, E. Duda, and H. Stobiecka. 1986. The change in disposition of plastids and mitochondria during microsporogenesis and sporogenesis in some higher plants. Acta Bot. Neerl. 35:209-215.

Russo, G. 2004. Clementine 'Angiulli': an early ripening mutation of the clementine 'Comune', p. 109. In: E. Mohamed (ed.). Program and Abstract, Intl. Soc. Citriculture, 10th Intl. Congr., Agadir, Morocco (abstr.).

Singh, H. 1978. Embryology of gymnosperms. Gebruder Bornträger, Berlin.

Ting, J.T.L., S.S.H. Wu, C. Ratnayake, and A.H.C. Huang. 1998. Constituents of the tapetosomes and elaioplasts in Brassica campestris tapetum and their degradation and retention during microsporogenesis. Plant J. 16:541-551.

Xie, C.T., Y.H. Yang, Y.L. Qiu, X.Y. Zhu, and H.Q. Tian. 2005. Cytochemical investigation of genic male-sterility in chinese cabbage. Sex. Plant Reprod. 18:75-80.

Yamamoto, M., N. Okudai, R. Matsumoto, and Y. Yamada. 1993. Seed number of hybrid plants in citrus with special reference to seed number of parents and pollen yield of individuals. J. Jpn. Soc. Hort. Sci. 61:757-762.

Yamamoto, M., R. Matsumoto, and Y. Yamada. 1995. Relationship between sterility and seedlessness in citrus. J. Jpn. Soc. Hort. Sci. 64:23-29.

Yamamoto, M., R. Matsumoto, N. Okudai, and Y. Yamada. 1997. Aborted anthers of citrus result from gene-cytoplasmic male sterility. Scientia Hort. 70:9-14.

Yang, X.P. 2002. [The investigation of 'Ougan' mandarin and its industrialization.] Citrus Zhejiang Province 19:5-8 (in Chinese).

Yi, H.L., X.X. Deng, R.X. Xia, G.H. Li, X.S. Fu, and Y. Tan. 2003. [A new cultivar 'Red Flesh Navel Orange']. Acta Hort. Sinica 30:115 (in Chinese)..

Zeng, Y.R., L.B. Zhang, J.P. Si, and X.H. Xu. 2005. [Preliminary study on seedlessness of seedless 'Ougan' mandarin.] J. Zhejiang For. College 22:359-362 (in Chinese).

Zhang, S.L. and D.D. Fernando. 2005. Structural, histochemical, and protein analysis of male reproductive development in willow. Sex. Plant Reprod. 18:37-46. 